\title{
Identification of Genetic Loci Jointly Influencing Schizophrenia Risk and the Cognitive Traits of Verbal-Numerical Reasoning, Reaction Time, and General Cognitive Function
}

\begin{abstract}
Olav B. Smeland, MD, PhD; Oleksandr Frei, PhD; Karolina Kauppi, PhD; W. David Hill, PhD; Wen Li, PhD; Yunpeng Wang, PhD; Florian Krull, PhD; Francesco Bettella, PhD; Jon A. Eriksen, PhD; Aree Witoelar, PhD; Gail Davies, PhD; Chun C. Fan, MD; Wesley K. Thompson, PhD; Max Lam, PhD; Todd Lencz, PhD; Chi-Hua Chen, PhD; Torill Ueland, PhD; Erik G. Jönsson, MD, PhD; Srdjan Djurovic, PhD; lan J. Deary, PhD; Anders M. Dale, PhD; Ole A. Andreassen, MD, PhD; for the NeuroCHARGE (Cohorts for Heart and Aging Research in Genomic Epidemiology) Cognitive Working Group
\end{abstract}

IMPORTANCE Schizophrenia is associated with widespread cognitive impairments. Although cognitive deficits are one of the factors most strongly associated with functional outcome in schizophrenia, current treatment strategies largely fail to ameliorate these impairments. To develop more efficient treatment strategies in patients with schizophrenia, a better understanding of the pathogenesis of these cognitive deficits is needed. Accumulating evidence indicates that genetic risk of schizophrenia may contribute to cognitive dysfunction.

OBJECTIVE To identify genomic regions jointly influencing schizophrenia and the cognitive domains of reaction time and verbal-numerical reasoning, as well as general cognitive function, a phenotype that captures the shared variation in performance across cognitive domains.

DESIGN, SETTING, AND PARTICIPANTS Combining data from genome-wide association studies from multiple phenotypes using conditional false discovery rate analysis provides increased power to discover genetic variants and could elucidate shared molecular genetic mechanisms. Data from the following genome-wide association studies, published from July 24, 2014, to January 17, 2017, were combined: schizophrenia in the Psychiatric Genomics Consortium cohort ( $n=79757$ [cases, 34486 ; controls, 45271 ]); verbal-numerical reasoning ( $n=36$ 035) and reaction time ( $n=111483)$ in the UK Biobank cohort; and general cognitive function in CHARGE (Cohorts for Heart and Aging Research in Genomic Epidemiology) $(n=53949)$ and COGENT (Cognitive Genomics Consortium) $(n=27888)$.

MAIN OUTCOMES AND MEASURES Genetic loci identified by conditional false discovery rate analysis. Brain messenger RNA expression and brain expression quantitative trait locus functionality were determined.

RESULTS Among the participants in the genome-wide association studies, 21 loci jointly influencing schizophrenia and cognitive traits were identified: 2 loci shared between schizophrenia and verbal-numerical reasoning, 6 loci shared between schizophrenia and reaction time, and 14 loci shared between schizophrenia and general cognitive function. One locus was shared between schizophrenia and 2 cognitive traits and represented the strongest shared signal detected (nearest gene TCF2O; chromosome 22q13.2), and was shared between schizophrenia ( $z$ score, $5.01 ; P=5.53 \times 10^{-7}$ ), general cognitive function ( $z$ score, -4.43 ; $\left.P=9.42 \times 10^{-6}\right)$, and verbal-numerical reasoning $\left(z\right.$ score, $\left.-5.43 ; P=5.64 \times 10^{-8}\right)$. For 18 loci, schizophrenia risk alleles were associated with poorer cognitive performance. The implicated genes are expressed in the developmental and adult human brain. Replicable expression quantitative trait locus functionality was identified for 4 loci in the adult human brain.

CONCLUSIONS AND RELEVANCE The discovered loci improve the understanding of the common genetic basis underlying schizophrenia and cognitive function, suggesting novel molecular genetic mechanisms.

JAMA Psychiatry. 2017;74(10):1065-1075. doi:10.1001/jamapsychiatry.2017.1986 Published online July 26, 2017. Corrected on August 16, 2017.
Supplemental content

Author Affiliations: Author affiliations are listed at the end of this article.

Group Information: The NeuroCHARGE Cognitive Working Group members are listed at the end of this article.

Corresponding Author: Olav B. Smeland, MD, PhD, Division of Mental Health and Addiction, Oslo University Hospital, Kirkeveien 166, 0424 Oslo, Norway(o.b.smeland@medisin.uio.no). 
S chizophrenia is a severe, chronic psychiatric disorder that ranks among the leading causes of disability worldwide. ${ }^{1}$ Although the diagnosis of schizophrenia is based on the presence of positive and negative symptoms, cognitive dysfunction is regarded as a core component of the disorder. ${ }^{2-4}$ Compared with healthy individuals, patients with schizophrenia display widespread cognitive impairments including deficits in learning, memory, processing speed, attention, and executive functioning. ${ }^{3,5,6}$ Cognitive dysfunction often precedes the onset of psychosis by several years ${ }^{7,8}$ and is an important factor associated with functional outcomes in schizophrenia. ${ }^{2,9}$ Despite this fact, current treatment strategies largely fail to ameliorate the cognitive deficits in patients with schizophrenia., ${ }^{2,4,10}$ To develop more efficient treatment strategies for patients with schizophrenia, a better understanding of the pathogenesis underlying these cognitive deficits is needed.

Accumulating evidence indicates that the genetic risk of schizophrenia may contribute to cognitive impairment. ${ }^{11}$ Unaffected relatives of patients with schizophrenia display cognitive deficits, ${ }^{12-14}$ and family and twin studies find that the genetic liabilities of schizophrenia and cognitive abilities covary. ${ }^{15,16}$ Further evidence comes from analyses of genomewide association study (GWAS) data. Polygenic risk scores based on GWAS data for schizophrenia are associated with decreased cognitive abilities in nonclinical cohorts, ${ }^{17-21}$ while polygenic risk for lower cognitive abilities is associated with increased likelihood of schizophrenia. ${ }^{18,19}$ Moreover, recent analyses of GWAS data estimated significant negative correlations between the genomic architectures of schizophrenia and different cognitive traits known to be affected in schizophrenia, including general cognitive function (GCF), verbalnumerical reasoning (VNR), and reaction time (RT) ${ }^{19,22-24}$ However, despite the robust evidence for a common genetic basis between cognitive dysfunction and schizophrenia, the specific gene variants jointly influencing schizophrenia and cognitive traits remain to be determined. ${ }^{11}$

Analyses of GWAS data have estimated single-nucleotide polymorphism (SNP)-based heritabilities of $33 \%$ for schizophrenia, ${ }^{25} 28 \%$ for GCF, ${ }^{26} 31 \%$ for VNR, ${ }^{27}$ and $11 \%$ for $\mathrm{RT}^{27}$ To date, 5 genome-wide significant lociare identified for $\mathrm{GCF}^{24,26}$ 3 loci for VNR, ${ }^{27}$ and 2 loci for RT, ${ }^{27}$ while more than 100 loci are identified for schizophrenia. ${ }^{28}$ However, despite the assembly of very large GWAS cohorts, the identified genome-wide significant loci explain only a small fraction of the heritability of these phenotypes. ${ }^{24,26-28}$ To improve discovery of genetic variants in polygenic human disorders, we have developed a conditional false discovery rate (CFDR) statistical approach that includes all available variants in 2 independent GWASs. ${ }^{29-31}$ The cFDR method enables combined analysis of GWAS data with increased power to discover overlapping genetic variants and could elucidate shared molecular genetic mechanisms. ${ }^{29-31}$ Using this approach, studies have identified shared loci between schizophrenia, ${ }^{32-35}$ Alzheimer disease, ${ }^{36}$ immune-related diseases, ${ }^{29,33,37}$ and associated phenotypes and substantially increased the number of identified risk loci. Here, we used the same statistical approach, taking advantage of several large GWASs, ${ }^{24,26-28}$ to identify common genetic variants shared between schizophrenia and VNR, RT, and GCF.

\section{Key Points}

Question What genetic loci jointly influence schizophrenia and cognitive function?

Findings In this analysis of genome-wide association studies on schizophrenia and cognitive traits in more than 250000 participants, 21 genomic regions were found to be shared between schizophrenia and cognitive traits.

Meaning The findings provide new insights into the common genetic basis underlying schizophrenia and cognitive function, suggesting novel molecular genetic mechanisms.

\section{Methods}

\section{Participant Samples}

We obtained GWAS results in the form of summary statistics ( $P$ values and $z$ scores). Data on schizophrenia were acquired from the Psychiatric Genomics Consortium $(\mathrm{n}=79757),{ }^{28}$ data on GCF were from the Cohorts for Heart and Aging Research in Genomic Epidemiology (CHARGE) $(n=53949)^{26}$ and the Cognitive Genomics Consortium (COGENT) $(n=27888),{ }^{24}$ and data on VNR ( $n=36035)$, and RT ( $n=111483)$ were from the UK Biobank.$^{27}$ These studies were published from July 24 , 2014 , to January 17, 2017. Details of the inclusion criteria, phenotype characteristics, and genotyping are described in the original publications. ${ }^{24,26-28}$ Overlapping cohorts $(n=7410)$ between CHARGE and COGENT were excluded from the COGENT GWAS ( $n=35298$ in the full cohort). ${ }^{24}$ Participants from the Betula Study and the Hunter Community Study were involved in both the schizophrenia GWAS ${ }^{28}$ and the GCF GWAS by CHARGE, ${ }^{26}$ thus possibly implicating that 611 participants used as controls in the schizophrenia GWAS contributed to the CHARGE GWAS. To avoid any potential bias, we excluded the Betula Study and Hunter Community Study cohorts $(\mathrm{n}=2558)$ from the schizophrenia data. All $P$ values were corrected for inflation using a genomic inflation control procedure. ${ }^{32,34,37}$ All GWASs performed and investigated in the present study were approved by the local ethics committees, and informed consent was obtained from all participants. Furthermore, the Norwegian Institutional Review Board for the South-East Norway Region has evaluated the current protocol and found that no additional institutional review board approval was needed because no individual data were used.

\section{Cognitive Phenotypes}

We analyzed adequately powered GWASs ${ }^{24,26,27}$ for cognitive phenotypes known to be affected in schizophrenia ${ }^{3,5,6}$ : GCF, RT, and VNR. General cognitive function accounts for approximately $40 \%$ to $50 \%$ of the variation across cognitive domains. ${ }^{24,26}$ For each cohort contributing to the GWAS metaanalyses on GCF by CHARGE ${ }^{26}$ and COGENT, ${ }^{24}$ the GCF phenotype was constructed using the first unrotated component extracted from a principal components analysis of the individual cognitive test scores. ${ }^{24,26}$ The RT test was a computerized "Snap" game, in which participants were to press a button as quickly as possible when symbols of 2 "cards" on a 
computer screen were matching. ${ }^{27}$ There were 8 experimental trials, of which 4 had matching symbols. Each participant's RT score was his or her mean time to press the button for these 4 matching trials. Verbal-numerical reasoning was measured using a 13-item test assessing verbal and arithmetical deduction. ${ }^{27}$ The test included 6 verbal and 7 numerical questions, all with multiple-choice answers, and had a total time limit of 2 minutes. For full details of each test, see the original publications. ${ }^{24,26,27}$

\section{Statistical Analysis}

To assess for pleiotropic enrichment, we constructed conditional quantile-quantile plots, which compare the association with a primary trait (eg, schizophrenia) across all SNPs and within SNP strata determined by their association with a secondary trait (eg, GCF), and provide a visual pattern of overlap in SNP associations. For given associated phenotypes A and $B$, pleiotropic enrichment of phenotype A with phenotype $B$ exists if the proportion of SNPs or genes associated with phenotype A increases as a function of increased association with phenotype B. The enrichment seen can be directly interpreted in terms of true discovery rate (1 - FDR). To identify shared loci between schizophrenia and cognitive traits, we used the cFDR framework. ${ }^{29,32-34,36,37}$ The cFDR is an extension of the standard FDR and incorporates information from GWAS summary statistics of a secondary phenotype to rerank the test statistics. We identified shared loci at a conjunctional FDR (conjFDR) less than .05, which is given by the maximum between the cFDRs for both phenotypes. ${ }^{38}$ The conjFDR analysis is a conservative approach requiring that loci exceed a cFDR significance threshold for 2 traits jointly. ${ }^{32,38}$ Because the cognitive traits investigated are not independent, we did not perform a Bonferroni correction. The risk loci were annotated to the closest gene. Given the long-range linkage disequilibrium (LD) within the extended major histocompatibility complex and its strong association with schizophrenia, ${ }^{28}$ we excluded SNPs in this region (genome build 19 location, 2565242933368333) and SNPs in LD ( $\left.r^{2}>0.1\right)$ with such SNPs before fitting the cFDR model. ${ }^{39}$ For details, see the eAppendix in the Supplement. The significance threshold for identification of shared loci was conjFDR < .05; for conditional loci, cFDR < .01; for gene set enrichment, FDR < .05; and for expression quantitative trait locus (eQTL) functionality, FDR $<.05$.

\section{Biological Context}

We evaluated the biological context of the identified genetic variants. First, we determined the distribution of messenger RNA expression in the developing and adult human brain using data from the Human Brain Transcriptome Project ${ }^{40}$ and The UK Brain Expression Consortium (UKBEC). ${ }^{41}$ Second, we assessed whether the conjunctional SNPs have brain eQTL functionality using Genotype-Tissue Expression (GTEx) ${ }^{42}$ and UKBEC (UK Brain Expression Consortium) ${ }^{41}$ data. Finally, we determined whether genes in the loci shared between schizophrenia and GCF were enriched for reconstituted versions of gene sets using the computational tool Data-driven Expression Prioritized Integration for Complex Traits (DEPICT). ${ }^{43}$ For details, see the eAppendix in the Supplement.

\section{Results}

We observed SNP enrichment for schizophrenia with VNR, RT, and GCF in the CHARGE and COGENT cohorts, indicating polygenic overlap between these phenotypes (Figure 1), in line with results of previous work. ${ }^{15,18-20,22-24}$ The reverse conditional quantile-quantile plots demonstrate consistent enrichment in associations with GCF, VNR, and RT as a function of schizophrenia associations (eFigure 1 in the Supplement). To increase discovery of SNPs associated with cognitive traits, we ranked GCF, VNR, and RT SNPs conditional on their genetic association with schizophrenia (cFDR). At cFDR less than .01, we identified 8 loci associated with GCF in the CHARGE cohort, 3 loci associated with GCF in the COGENT cohort, 5 loci associated with VNR, and 4 loci associated with RT (eTable 1 in the Supplement). Figure 2 shows cFDR Manhattan plots for GCF, VNR, and RT conditional on schizophrenia, showing all SNPs with a cFDR less than .01 within an LD block in relation to their chromosomal location. The figures demonstrate the increased power for SNP discovery gained by conditioning on association with schizophrenia.

To provide a comprehensive, unselected map of shared loci between schizophrenia and GCF, VNR, and RT, we performed a conjFDR analysis (Figure 3). Based on a conjFDR less than .05, we identified 21 independent genetic loci shared between schizophrenia and cognitive traits. Specifically, we identified 14 loci shared between schizophrenia and GCF in the CHARGE cohort, 2 loci shared between schizophrenia and VNR, and 6 loci shared between schizophrenia and RT, on a total of 13 chromosomes (1 locus was shared between schizophrenia and 2 cognitive traits) (Table). ${ }^{26-28}$ We detected no loci shared between schizophrenia and GCF in the COGENT cohort. The strongest shared signal detected (nearest gene TCF2O [OMIM 603107]; chromosome 22q13.2) was shared between schizophrenia $\left(z\right.$ score, 5.01; $\left.P=5.53 \times 10^{-7}\right)$, GCF ( $z$ score, -4.43 ; $\left.P=9.42 \times 10^{-6}\right)$, and VNR $\left(z\right.$ score, $\left.-5.43 ; P=5.64 \times 10^{-8}\right)$, demonstrating the importance of the locus for brain function. Thirteen of 21 conjunctional loci have $P$ values less than .05 in at least 2 cognitive traits. To visualize the shared loci, we constructed a conjFDR Manhattan plot (Figure 3). All SNPs without pruning are shown, and the strongest signal in each LD block is encircled in black. The enlarged data points represent the SNPs at conjFDR less than .05, whereas the small points represent other SNPs. On the basis of 1000 Genomes Project LD structure, significant SNPs identified by conjFDR less than .05 were clustered into LD blocks at the LD level of $r^{2}>0.1$. These blocks are numbered in the Table. ${ }^{26-28}$ Any block may contain more than 1 SNP. Genes close to each locus were obtained from the National Center for Biotechnology Information gene database (https://www.ncbi.nlm.nih.gov).

We evaluated the directionality of allelic effects in the loci shared between schizophrenia and cognitive traits by investigating their $z$ scores (Table). ${ }^{26-28}$ For 10 loci shared between schizophrenia and GCF, we found opposite effect directions in the phenotypes, while rs12521503 (SSBP2 [OMIM 607389]) showed concordant effect directions. Owing to T/A polymorphisms, the effect directions were ambiguous for rs13024343 
Figure 1. Polygenic Overlap Between Schizophrenia (SCZ) and General Cognitive Function (GCF), Reaction Time (RT), and Verbal-Numerical Reasoning (VNR)

\begin{tabular}{|c|c|}
\hline $\begin{array}{l}\text { All SNPs } \\
P<.10 \text { cognitive trait }\end{array}$ & $\begin{array}{l}P<.01 \text { cognitive trait } \\
P<.001 \text { cognitive trait }\end{array}$ \\
\hline
\end{tabular}

A SCZ | GCF CHARGE

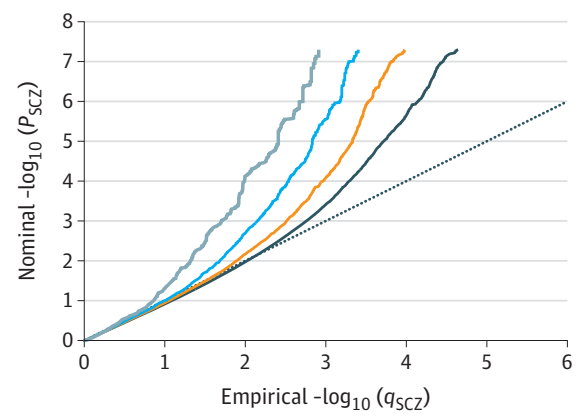

C SCZ IRT

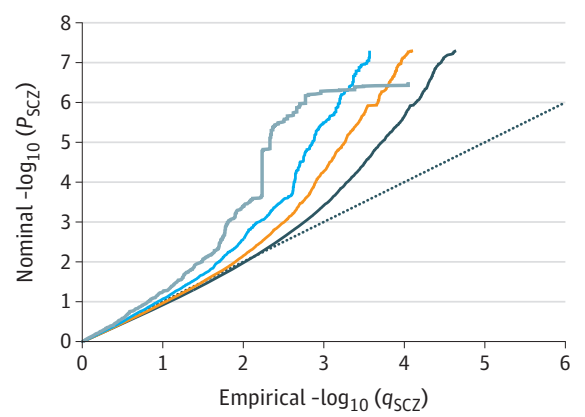

B SCZ | GCF COGENT

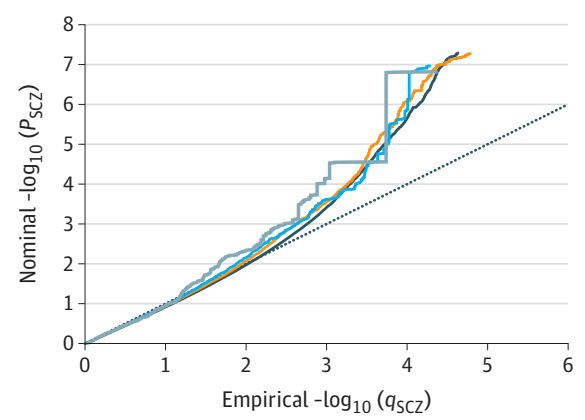

D SCZ IVNR

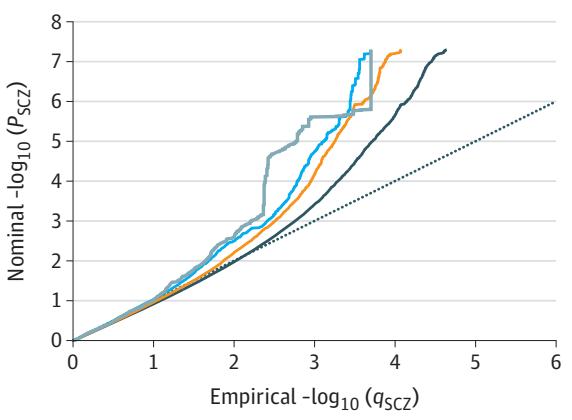

Conditional quantile-quantile plots of nominal vs empirical $-\log _{10} P$ values (corrected for inflation) in SCZ below the standard genome-wide association study threshold of $P<5 \times 10^{-8}$ as a function of significance of association with GCF in CHARGE (Cohorts for Heart and Aging Research in Genomic Epidemiology) (A), GCF in COGENT (Cognitive Genomics Consortium) (B), RT (C), and VNR (D), at the level of $-\log _{10}(P) \geq 1,-\log _{10}(P) \geq 2$, and $-\log _{10}(P) \geq 3$, corresponding to $P \leq .10, P \leq .01$, and $P \leq .001$, respectively. The dashed lines indicate the null hypothesis.
(CTNNA2 [OMIM 114025]), rs1545424 (TBC1D5 [OMIM 615740]), and rs12253987 (NEURL [OMIM 603804]). Singlenucleotide polymorphisms in LD with rs13024343 show concordant associations in schizophrenia and GCF, while SNPs in LD with rs1545424 and rs12253987 show inverse associations in the phenotypes (eTable 2 in the Supplement). Both loci shared between schizophrenia and VNR showed opposite effect directions in the phenotypes. For loci shared between schizophrenia and RT, 4 showed concordant associations in the phenotypes (ie, the schizophrenia risk alleles are associated with slower RT), while rs67338739 (KCNJ3 [OMIM 601534]) showed opposite effect directions. The effect directions were ambiguous for rs7857165 (PHF2 [OMIM 604351]; T/A polymorphism). Single-nucleotide polymorphisms in LD with rs7857165 show concordant associations in schizophrenia and RT (eTable 2 in the Supplement). The overall negative correlation between schizophrenia risk and cognitive performance was consistent among shared loci at conjFDR less than .10 (eTable 3 in the Supplement). Of note, identification of loci with conjFDR less than .10 implicated potential overlapping associations between schizophrenia and GCF located within the major histocompatibility complex region (eTable 3 in the Supplement). However, this subthreshold finding must be interpreted cautiously given the complex LD in this region.

Next, we determined the messenger RNA expression distribution in the human brain for genes implicated in the conjFDR analysis. Expression data provided by $\mathrm{UKBEC}^{41}$ demon- strate that the identified genes are globally expressed in the adult human brain (eFigure 2 in the Supplement). Data for RNA gene DQ584120 (GenBank DQ584120.1) were not available in this data set. Expression data from the Human Brain Transcriptome Project ${ }^{40}$ show that the genes are globally expressed in the developing and adult human brain (eFigure 3 in the Supplement). Data for ZSWIM6 (OMIM 615951) and $D Q 584120$ were not available in this data set. ${ }^{40}$ We further investigated the eQTL functionality of the identified conjunctional loci. Using GTEx ${ }^{42}$ data, we identified significant eQTL associations for 5 SNPs in human brain tissue (eTable 4 in the Supplement). We assessed the replicability of these brainspecific eQTLs using UKBEC ${ }^{41}$ data and identified significant replicable eQTL functionality for the following 4 SNPs: rs12993822 for KCNJ3 in the cerebellum, rs4282054 for GNL3 (OMIM 608011) in the frontal cortex and cerebellum, rs524908 for STRC (OMIM 606440) in the frontal cortex, and rs134873 for both CYP2D6 (OMIM 124030) in several brain regions and for NAGA (OMIM 104170) in the cerebellum and frontal cortex (eTable 5 in the Supplement). Not all brain regions were jointly examined by GTEx ${ }^{42}$ and UKBEC. ${ }^{41}$

The DEPICT analysis ${ }^{43}$ revealed multiple gene sets enriched for genes in loci shared between schizophrenia and GCF. However, the results did not remain significant after correction for multiple comparisons, which may reflect the relatively sparse number of analyzed loci and/or the subtlety of the biological signal. The top-ranked gene sets were "NFYA 
A GCF CHARGE conditioned on SCZ

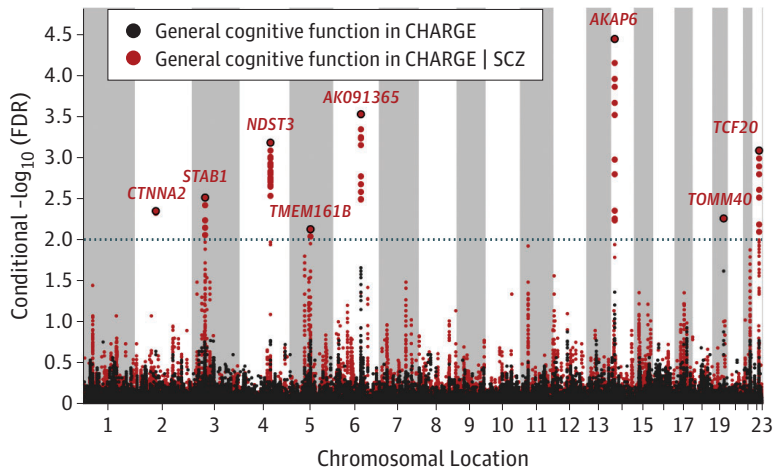

C Reaction time conditioned on SCZ

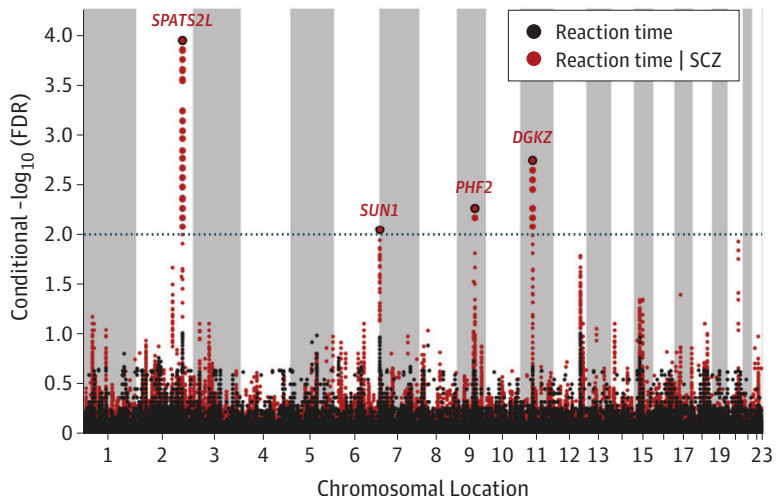

A, General cognitive function in CHARGE (Cohorts for Heart and Aging Research in Genomic Epidemiology) conditioned on schizophrenia (SCZ). B, General cognitive function in COGENT (Cognitive Genomics Consortium) conditioned on SCZ. C, Reaction time conditioned on SCZ. D, Verbal-numerical reasoning conditioned on SCZ. Unconditioned FDR values are shown in black, CFDR values
B GCF COGENT conditioned on SCZ

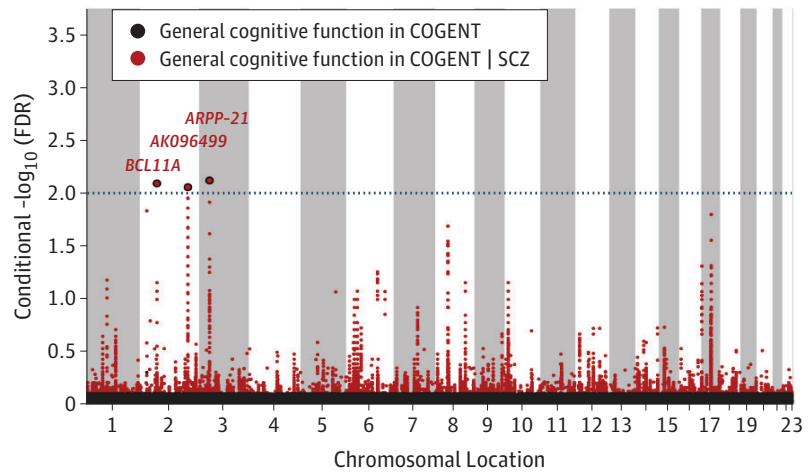

D Verbal-numerical reasoning conditioned on SCZ

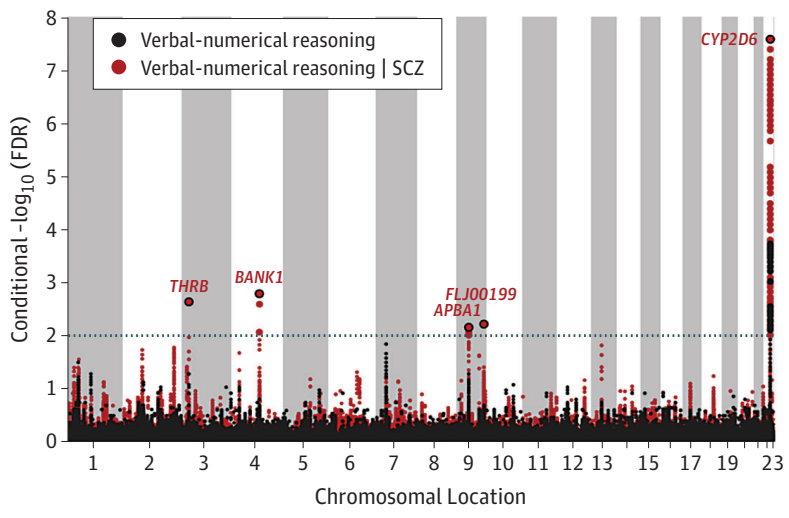

in red. Single-nucleotide polymorphisms (SNPs) with conditional - $\log _{10}$ (FDR) higher than 2.0 (horizontal dotted line) (ie, cFDR < .01) are shown with large points. A black line around the large points indicates the most significant SNP in each linkage disequilibrium block; this SNP is annotated with the closest gene.
(OMIM 189903) subnetwork," "increased neuron apoptosis," and "chromatin remodeling complex" (eFigure 4 in the Supplement). NFYA encodes the sequence-specific DNA-binding subunit of the transcription factor NF-Y, ${ }^{45}$ a key regulator of differentiation in various proliferative cells. NF-Y is also active in mature neurons and may be involved in neurodegeneration. ${ }^{46}$

\section{Discussion}

In the present study, we analyzed GWAS data using cFDR analysis and identified 21 genetic variants jointly influencing risk of schizophrenia and the cognitive traits of GCF, VNR, and RT. We found that genetic enrichment in cognitive traits based on SNP association with schizophrenia results in improved statistical power for gene discovery and increased gene discovery for these cognitive traits. Most of the loci found to be shared between schizophrenia and cognitive traits (18 of 21) show a negative correlation between risk of schizophrenia and cognitive performance, in line with the observed cognitive dysfunction in schizophrenia ${ }^{3,5,6}$ and prior genetic studies. ${ }^{15,18-20,22-24,44}$ Altogether, this study provides new insights into the common genetic basis of schizophrenia and cognitive traits, suggesting novel molecular genetic mechanisms.

Recent studies applying LD score regression reported significant negative correlations between the genomic architectures of schizophrenia and GCF, ${ }^{23,24} \mathrm{VNR},{ }^{22}$ and RT. ${ }^{22}$ Here, we were able to dissect these coheritabilities by identifying multiple gene loci inversely associated with schizophrenia and these cognitive traits (Table). ${ }^{26-28}$ A total of 13 of 21 loci shared between schizophrenia and cognitive traits have a $P$ value less than .05 in at least 2 cognitive traits, demonstrating consistent associations of the identified loci across cognitive domains (Table) ${ }^{26-28}$ and supporting the credibility of the cFDR approach. Among the shared loci, 13 are novel for schizophrenia. ${ }^{28}$ The overall negative association between allelic effect directions for schizophrenia and cognitive performance was consistent among conjunctional loci identified at a relaxed significance threshold (conjFDR $<.10$; eTable 3 in the Supplement). The low SNP enrichment observed with GCF in the COGENT cohort is likely attributable to the smaller sample 
Figure 3. Conjunctional False Discovery Rate (FDR) Manhattan Plot of Conjunctional - $\log _{10}$ (FDR) Values for Schizophrenia (SCZ) and General Cognitive Function, Verbal-Numerical Reasoning, or Reaction Time

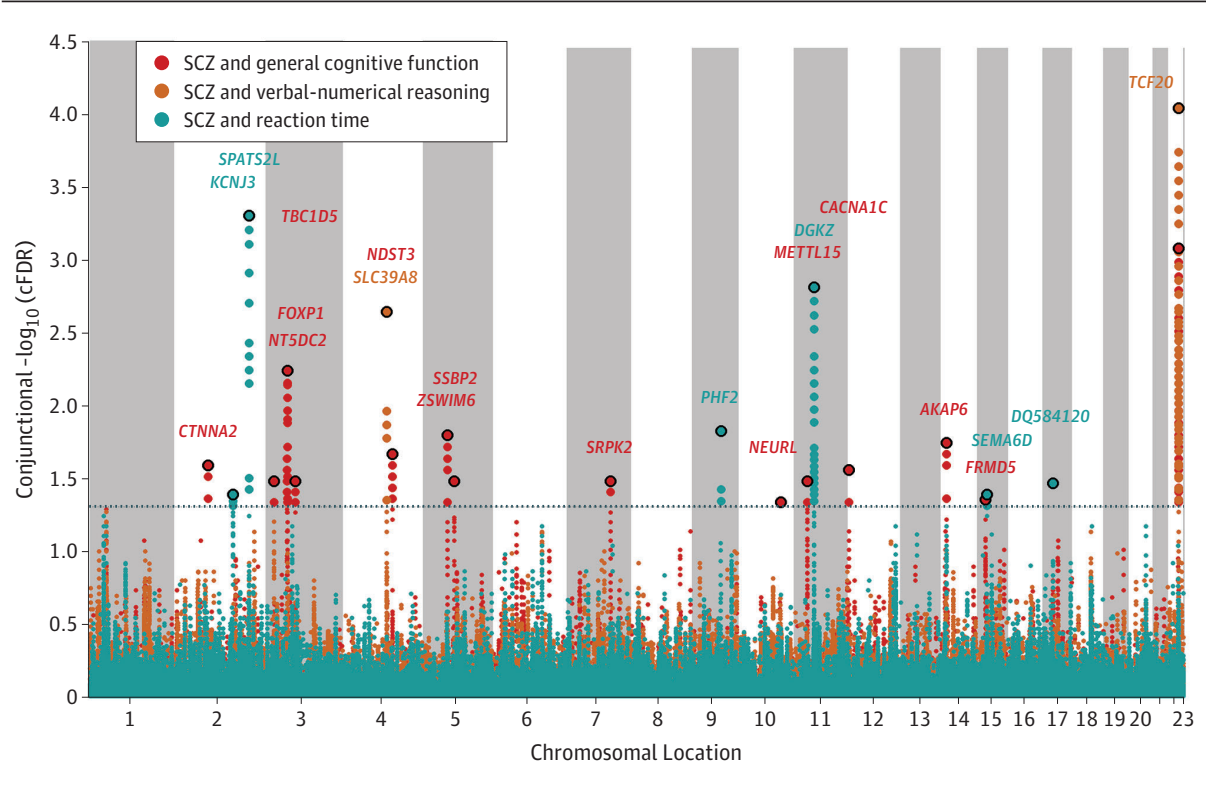

Single-nucleotide polymorphisms (SNPs) with conjunctional $-\log _{10}$ (FDR) $>1.3$, ie, conjunctional FDR $<.05$, are shown with enlarged data points. A black circle around the enlarged data points indicates the most significant SNP in each linkage disequilibrium block; this SNP was annotated with the closest gene, which is listed above the symbols in each locus. The localization of the conjunctional loci is shown; further details are provided in the Table. size of this cohort $(n=27888)^{24}$ compared with the CHARGE cohort $(n=53949) .{ }^{26}$ We identified 3 loci with the same effect directions in schizophrenia and cognitive skills (rs13024343 and rs12521503 shared between schizophrenia and GCF, and rs67338739 shared between schizophrenia and RT), demonstrating that some schizophrenia risk loci may increase the likelihood of better cognitive performance. These results emphasize the complexity of the shared genetic effects influencing schizophrenia and cognitive function. Although many schizophrenia risk loci may not affect cognitive function, the findings may shed light on the positive genetic correlation between schizophrenia and measures of creativity ${ }^{47}$ and education, ${ }^{35}$ and why some patients with schizophrenia exhibit normal cognitive skills. ${ }^{48}$ Given that schizophrenia typically manifests in adolescence, while GCF, VNR, and RT were measured in adults older than 40 years of age, ${ }^{26,27}$ the conjunctional loci appear to influence brain function across a person's life span. This finding is supported by the messenger RNA expression data showing that the implicated genes are globally expressed in the developing and adult human brain (eFigure 3 in the Supplement), and complies with previous work reporting genetic overlap between schizophrenia and cognitive abilities in middle and older age. ${ }^{17,20,23}$ Cognitive performance of participants in the CHARGE ${ }^{26}$ and UK Biobank GWASs $^{27}$ may have been susceptible to age-associated cognitive decline or subclinical neurodegenerative conditions. ${ }^{26}$ Moreover, the genetics underlying cognitive variation in healthy individuals might differ across their life spans. Given that schizophrenia manifests in adolescence and cognitive decline often precedes the onset of psychosis by many years, 7,8 an evaluation of shared genetic effects between schizophrenia and cognitive function measured in younger people is warranted.
The strongest signal of shared genetic effects between schizophrenia and cognitive traits was detected on chromosome 22q13.2, at a locus that contains many genes (nearest gene TCF2O; Table ${ }^{26-28}$ ). This locus was shared between schizophrenia, GCF, and VNR and was genome-wide significant in the primary GWASs on schizophrenia ${ }^{28}$ and VNR, ${ }^{27}$ but is a novel finding for GCF. TCF2O encodes a widely expressed transcriptional coregulator, and TCF2O mutations are associated with autism and intellectual disability. ${ }^{49}$ Using data from $\mathrm{GTEx}^{42}$ and UKBEC, ${ }^{41}$ we identified replicable eQTL functionality of the 22q13.2 locus for genes CYP2D6 and NAGA in several human brain regions (eTables 4 and 5 in the Supplement). The allele G of rs134873, which is associated with increased risk of schizophrenia and lower GCF and VNR scores, was associated with higher expression of NAGA and lower expression of CYP2D6. NAGA encodes a lysosomal enzyme that modifies glycoconjugates, ${ }^{50}$ and CYP2D 6 encodes a cytochrome $\mathrm{P} 450$ enzyme that metabolizes a broad range of drugs, including antipsychotics, ${ }^{51}$ and may also be involved in the metabolism of neurotransmitters, including serotonin and dopamine. ${ }^{52}$ Another notable locus shared between schizophrenia and GCF is the intronic variant within CACNA1C (OMIM 114205) (rs2238057). Genetic variation in CACNA1C is robustly implicated in schizophrenia, ${ }^{25,28,53}$ and it is associated with cognitive impairment in patients with schizophrenia and in healthy individuals. ${ }^{54,55}$ We also found that a locus at AKAP6 (OMIM 604691) (rs12885467, 3' UTR variant) jointly influences schizophrenia and GCF. This locus reached genomewide significance for GCF in the CHARGE cohort ${ }^{26}$ and is replicated across all cognitive traits. The number of shared loci identified here is consistent with results of previous conjFDR analyses $^{32,34,35}$ and depends on both the extent of genetic overlap between traits and the power of the investigated GWASs. 


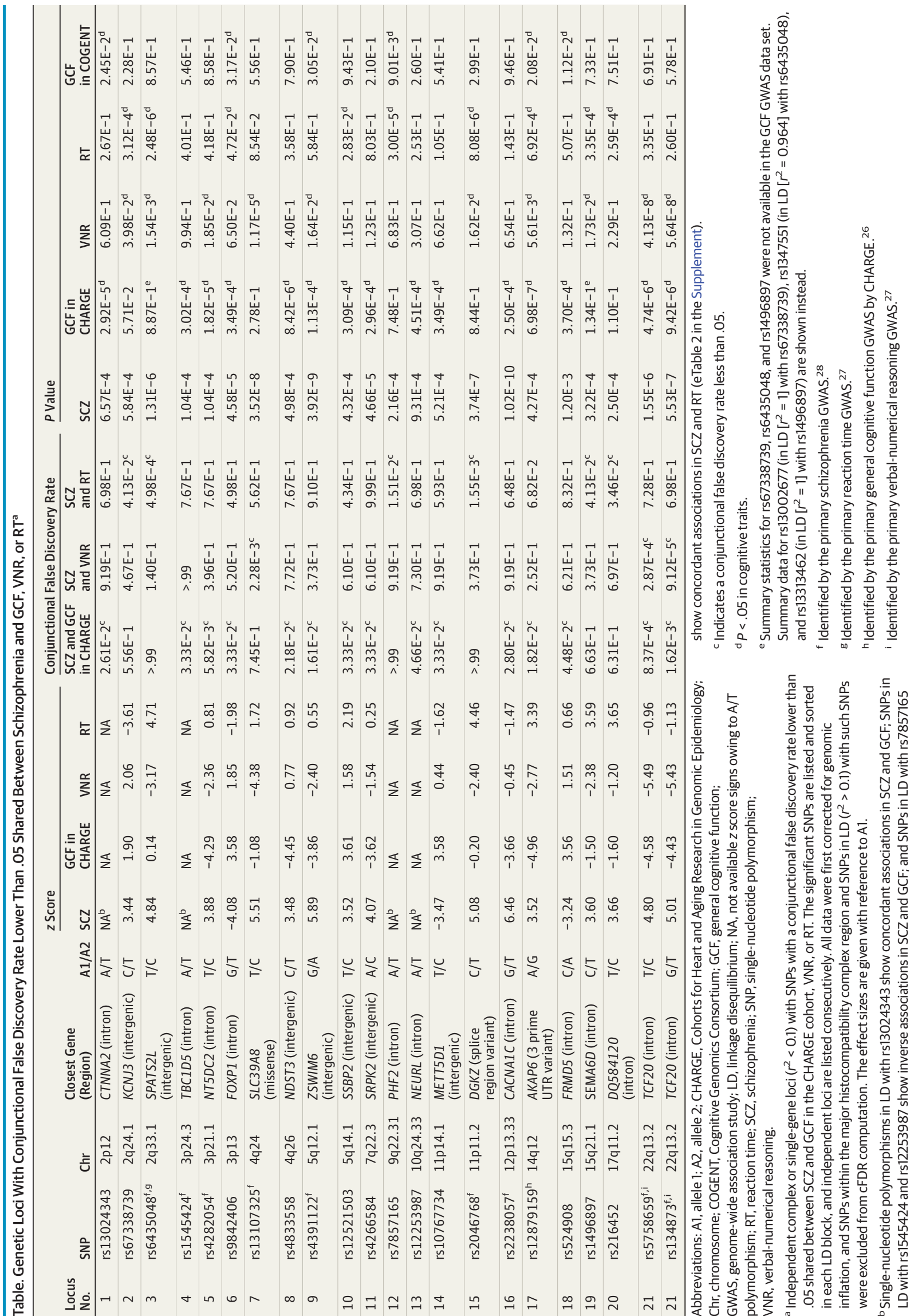


More shared loci between schizophrenia and cognitive traits are expected to be uncovered when larger GWAS samples are available.

\section{Limitations}

As with all GWAS findings, any SNP represents through LD a genomic region including potentially many causal SNPs. Hence, further studies are required to determine the true causal variants underlying the shared associations detected here, and whether the same causal variants are involved in schizophrenia and cognitive traits. ${ }^{56}$

\section{Conclusions}

We were able to increase discovery of genetic loci jointly influencing schizophrenia and cognitive traits using the cFDR approach. The findings provide new insights into the coheritability underlying schizophrenia and cognitive traits beyond their known genetic correlation. ${ }^{15,18-20,23,24,44}$ The discovered loci can be used as resources and to guide further efforts to disentangle the neurobiological basis underlying schizophrenia and cognitive function.

\section{ARTICLE INFORMATION}

Accepted for Publication: May 30, 2017.

Published Online: July 26, 2017. doi:10.1001/jamapsychiatry.2017.1986

Correction: This article was corrected on August 16, 2017, for a typographical error in the Abstract.

Author Affiliations: Norwegian Centre for Mental Disorders Research, KG Jebsen Centre for Psychosis Research, Institute of Clinical Medicine, University of Oslo, Oslo, Norway (Smeland, Frei, Li, Wang, Krull, Bettella, Eriksen, Witoelar, Ueland, Jönsson, Andreassen); Norwegian Centre for Mental Disorders Research, Division of Mental Health and Addiction, Oslo University Hospital, Oslo, Norway (Smeland, Jönsson, Andreassen); Department of Neuroscience, University of California San Diego, La Jolla (Smeland, Dale); Department of Radiology, University of California San Diego, La Jolla (Kauppi, Wang, Fan, Chen, Dale); Center for Multimodal Imaging and Genetics, University of California San Diego, La Jolla (Kauppi, Wang, Chen, Dale); Department of Radiation Sciences, Umeå University, Umeå, Sweden (Kauppi); Centre for Cognitive Ageing and Cognitive Epidemiology, University of Edinburgh, Edinburgh, United Kingdom (Hill, Davies, Deary); Department of Psychology, University of Edinburgh, Edinburgh, United Kingdom (Hill, Davies, Deary); Department of Cognitive Science, University of California, San Diego, La Jolla (Fan); Department of Family Medicine and Public Health, University of California, San Diego, La Jolla (Thompson); Institute of Biological Psychiatry, Roskilde, Denmark (Thompson); Institute of Mental Health, Singapore (Lam); Division of Psychiatry Research, Zucker Hillside Hospital, Glen Oaks, New York (Lencz) Center for Psychiatric Neuroscience, Feinstein Institute for Medical Research, Manhasset, New York (Lencz); Department of Psychiatry, Hofstra Northwell School of Medicine, Hempstead, New York (Lencz); Department of Psychology, University of Oslo, Olso, Norway (Ueland); Department of Clinical Neuroscience, Centre for Psychiatric Research, Karolinska Institutet, Stockholm, Sweden (Jönsson); Department of Medical Genetics, Oslo University Hospital, Oslo, Norway (Djurovic); NORMENT, KG Jebsen Centre for Psychosis Research, Department of Clinical Science, University of Bergen, Bergen, Norway (Djurovic); Department of Psychiatry, University of California, San Diego, La Jolla (Dale)

Author Contributions: Drs Smeland and Frei had full access to all the data in the study and take responsibility for the integrity of the data and the accuracy of the data analysis.

Study concept and design: Smeland, Kauppi, Dale, Andreassen.
Acquisition, analysis, or interpretation of data: All authors.

Drafting of the manuscript: Smeland. Critical revision of the manuscript for important intellectual content: All authors.

Statistical analysis: Smeland, Frei, Kauppi, Li, Wang, Krull, Eriksen, Witoelar, Fan, Thompson, Lencz, Dale. Obtained funding: Smeland, Wang, Thompson, Chen, Deary, Dale, Andreassen.

Administrative, technical, or material support: $\mathrm{Li}$ Wang, Bettella, Lencz, Ueland, Djurovic, Deary, Dale, Andreassen.

Study supervision: Smeland, Deary, Dale, Andreassen.

Conflict of Interest Disclosures: Dr Andreassen reported receiving a speaker's honorarium from Lundbeck. No other disclosures were reported.

Funding/Support: This study was supported by grants R01GM104400, NSO57198, and EB00790 from the National Institutes of Health; grant R01MH100351 from the National Institute of Mental Health; grant U24DA041123 from the National Institutes of Health, National Institute on Drug Abuse, and National Cancer Institute; grants 229129. 213837, 248778, 223273, and 249711 from the Research Council of Norway; grants 2016-064 2017-004, and 2017-112 from the South-East Norway Regional Health Authority; grant SKGJ-2011-36 from KG Jebsen Stiftelsen; grant MR/K026992/1 from the UK Biotechnology and Biological Sciences Research Council and Medical Research Council; and the Research Council of Norway through a FRIPRO Mobility Grant, contract No. 251134 (Dr. Wang). The FRIPRO Mobility grant scheme (FRICON) is co-funded by the European Union's Seventh Framework Programme for research, technological development and demonstration under Marie Curie grant agreement No. 608695

Role of the Funder/Sponsor: The funding sources had no role in the design and conduct of the study: collection, management, analysis, and interpretation of the data; preparation, review, or approval of the manuscript; and decision to submit the manuscript for publication.

Group Information: The NeuroCHARGE Cognitive Working Group was part of the collaboration responsible for this work. Its members are Gail Davies, PhD (Centre for Cognitive Ageing and Cognitive Epidemiology, University of Edinburgh, Edinburgh, UK; Department of Psychology, University of Edinburgh, Edinburgh, UK); Nicola Armstrong, PhD (School of Mathematics and Statistics, University of Sydney, Sydney, NSW, Australia); Joshua C. Bis, PhD (Cardiovascular Health Research Unit, Department of Medicine, University of Washington, Seattle); Jan Bressler, PhD (Human Genetics Center, School of Public Health, University of Texas Health Science Center at Houston); Vincent
Chouraki, MD, PhD (Inserm-UMR744, Institut Pasteur de Lille, Unité d'Epidémiologie et de Santé Publique, Lille, France; Department of Neurology, Boston University School of Medicine, Boston, MA): Sudheer Giddaluru, PhD (K.G. Jebsen Centre for Psychosis Research and the Norwegian Centre for Mental Disorders Research [NORMENT],

Department of Clinical Science, University of Bergen, Bergen, Norway; Dr Einar Martens Research Group for Biological Psychiatry, Center for Medical Genetics and Molecular Medicine, Haukeland University Hospital, Bergen, Norway); Edith Hofer, $\mathrm{PhD}$ (Department of Neurology, Medical University of Graz, Graz, Austria; Institute for Medical Informatics, Statistics and Documentation, Medical University of Graz, Graz, Austria); Carla A. Ibrahim-Verbaas, MD, PhD (Department of Neurology, Erasmus University Medical Center، Rotterdam, the Netherlands; Genetic Epidemiology Unit, Department of Epidemiology, Erasmus University Medical Center, Rotterdam, the Netherlands); Mirna Kirin, PhD (Centre for Population Health Sciences, University of Edinburgh, Edinburgh, UK); Jari Lahti, PhD (Institute of Behavioural Sciences, University of Helsinki, Helsinki, Finland; Folkhälsan Research Centre, Helsinki, Finland); Sven J. van der Lee, MD (Genetic Epidemiology Unit, Department of Epidemiology, Erasmus University Medical Center, Rotterdam, the Netherlands); Stephanie Le Hellard, PhD (K.G. Jebsen Centre for Psychosis Research and the Norwegian Centre for Mental Disorders Research [NORMENT], Department of Clinical Science, University of Bergen, Bergen, Norway; Dr Einar Martens Research Group for Biological Psychiatry, Center for Medical Genetics and Molecular Medicine, Haukeland University Hospital, Bergen, Norway); Tian Liu, PhD (Max Planck Institute for Human Development, Berlin, Germany; Max Planck Institute for Molecular Genetics, Berlin, Germany); Riccardo E. Marioni, PhD (Centre for Cognitive Ageing and Cognitive Epidemiology, University of Edinburgh, Edinburgh, UK; Medical Genetics Section, University of Edinburgh Centre for Genomic and Experimental Medicine, Institute of Genetics and Molecular Medicine, Western General Hospital, Edinburgh, UK; Queensland Brain Institute, The University of Queensland, Brisbane, QLD, Australia); Christopher Oldmeadow, PhD (Hunter Medical Research Institute and Faculty of Health, University of Newcastle, Newcastle, NSW, Australia); Iris Postmus, PhD (Department of Gerontology and Geriatrics, Leiden University Medical Center, Leiden, the Netherlands; Netherlands Consortium for Healthy Ageing, Leiden, the Netherlands); Albert V. Smith, PhD (Icelandic Heart Association, Kopavogur, Iceland; Faculty of Medicine, University of Iceland, Reykjavik, Iceland); Jennifer A. Smith, PhD (Department of Epidemiology, University of Michigan, Ann Arbor); Anbupalam Thalamuthu, PhD 
(Centre for Healthy Brain Ageing, School of Psychiatry, University of New South Wales, Sydney, NSW, Australia); Russell Thomson, PhD (Menzies Research Institute, Hobart, Tasmania); Veronique Vitart, PhD (MRC Human Genetics Unit, Institute of Genetics and Molecular Medicine, University of Edinburgh, Edinburgh, UK); Jing Wang, PhD (Framingham Heart Study, Framingham, MA Department of Biostatistics, Boston University School of Public Health, Boston, MA); Lei Yu, PhD (Rush Alzheimer's Disease Center, Rush University Medical Center, Chicago, IL); Lina Zgaga, PhD (Department of Public Health and Primary Care, Trinity College Dublin, Dublin, Ireland; Andrija Stampar School of Public Health, Medical School University of Zagreb, Zagreb, Croatia); Wei Zhao, $\mathrm{PhD}$ (Department of Epidemiology, University of Michigan, Ann Arbor); Ruth Boxall, MSc (Medical Genetics Section, University of Edinburgh Centre for Genomic and Experimental Medicine, Institute of Genetics and Molecular Medicine, Western General Hospital, Edinburgh, UK); Sarah E. Harris, PhD (Centre for Cognitive Ageing and Cognitive Epidemiology, University of Edinburgh, Edinburgh، UK; Medical Genetics Section, University of Edinburgh Centre for Genomic and Experimental Medicine, Institute of Genetics and Molecular Medicine, Western General Hospital, Edinburgh, UK); W. David Hill, PhD (Department of Psychology, University of Edinburgh, Edinburgh, UK); David C. Liewald, BSc (Centre for Cognitive Ageing and Cognitive Epidemiology, University of Edinburgh Edinburgh, UK); Michelle Luciano, PhD (Centre for Cognitive Ageing and Cognitive Epidemiology, University of Edinburgh, Edinburgh, UK Department of Psychology, University of Edinburgh, Edinburgh, UK); Hieab H. Adams, MSc (Department of Epidemiology, Erasmus University Medical Center, Rotterdam, the Netherlands; Netherlands Consortium for Healthy Ageing, Leiden, the Netherlands); David Ames, MD (National Ageing Research Institute, Royal Melbourne Hospital, Melbourne, VIC, Australia; Academic Unit for Psychiatry of Old Age

St George's Hospital, University of Melbourne, Kew Australia); Najaf Amin, PhD (Genetic Epidemiology Unit, Department of Epidemiology, Erasmus University Medical Center, Rotterdam, the Netherlands; Netherlands Consortium for Healthy Ageing, Leiden, the Netherlands); Philippe Amouyel, MD, PhD (Inserm-UMR744, Institut Pasteur de Lille, Unité d'Epidémiologie et de Santé Publique, Lille, France); Amelia A. Assareh, PhD (Centre for Healthy Brain Ageing, School of Psychiatry, University of New South Wales, Sydney, NSW, Australia); Rhoda $\mathrm{Au}, \mathrm{PhD}$ (Department of Neurology, Boston University School of Medicine, Boston, MA; Framingham Heart Study, Framingham, MA); James T. Becker, PhD (Department of Neurology, University of Pittsburgh, Pittsburgh, PA; Department of Psychiatry, University of Pittsburgh, Pittsburgh, PA; Department of Psychology, University of Pittsburgh, Pittsburgh, PA); Alexa Beiser, PhD (Department of Neurology, Boston University School of Medicine, Boston, MA; Framingham Heart Study, Framingham, MA; Department of Biostatistics, Boston University School of Public Health, Boston, MA); Claudine Berr, MD, PhD (Inserm, U106, Montpellier, France; Université Montpellier I, Montpellier, France); Lars Bertram, MD (Max Planck Institute for Molecular Genetics, Berlin, Germany; Faculty of Medicine, School of Public Health, Imperial College, London, UK); Eric Boerwinkle, PhD (Human Genetics Center, School of Public Health, University of Texas Health Science Center at Houston; Brown Foundation Institute of Molecular Medicine for the Prevention of
Human Diseases, University of Texas Health Science Center at Houston; Human Genome Sequencing Center, Baylor College of Medicine, Houston, TX); Brendan M. Buckley, PhD (Department of Pharmacology and Therapeutics, University College Cork, Cork, Ireland); Harry Campbell, MD (Centre for Population Health Sciences, University of Edinburgh, Edinburgh, UK); Janie Corley, PhD (Department of Psychology, University of Edinburgh, Edinburgh, UK); Philip L. De Jager, MD, PhD (Program in Translational NeuroPsychiatric Genomics, Department of Neurology, Brigham and Women's Hospital, Boston, MA; Harvard Medical School, Boston, MA; Program in Medical and Population Genetics, Broad Institute, Cambridge, MA); Carole Dufouil, PhD (Inserm U708. Neuroepidemiology, Paris, France; Inserm U897, Université Bordeaux Segalen, Bordeaux, France); Johan G. Eriksson, MD, PhD (Folkhälsan Research Centre, Helsinki, Finland; National Institute for Health and Welfare, Helsinki, Finland; Department of General Practice and Primary Health Care, University of Helsinki, Helsinki, Finland; Unit of General Practice, Helsinki University Central Hospital, Helsinki, Finland); Thomas Espeseth, PhD (K.G. Jebsen Centre for Psychosis Research, Norwegian Centre For Mental Disorders Research [NORMENT], Division of Mental Health and Addiction, Oslo University Hospital and Institute of Clinical Medicine, University of Oslo, Oslo, Norway; Department of Psychology, University of Oslo, Oslo Norway): Jessica D. Faul, PhD (Survey Research Center, Institute for Social Research, University of Michigan, Ann Arbor); Ian Ford, PhD (Robertson Center for Biostatistics, Glasgow, UK); Generation Scotland (Generation Scotland, University of Edinburgh Centre for Genomic and Experimental Medicine, Institute of Genetics and Molecular Medicine, Western General Hospital, Edinburgh, UK); Rebecca F. Gottesman, MD, PhD (Department of Neurology, Johns Hopkins University School of Medicine, Baltimore, MD); Michael E. Griswold, PhD (Center of Biostatistics and Bioinformatics, University of Mississippi Medical Center, Jackson); Vilmunder Gudnason, MD, PhD (Icelandic Heart Association, Kopavogur, Iceland; Faculty of Medicine, University of Iceland, Reykjavik, Iceland); Tamara B. Harris, MD (Intramural Research Program National Institutes on Aging, National Institutes of Health, Bethesda, MD); Gerardo Heiss, MD, PhD (Department of Epidemiology, University of North Carolina Gillings School of Global Public Health, Chapel Hill); Albert Hofman, MD, PhD (Department of Epidemiology, Erasmus University Medical Center, Rotterdam, the Netherlands; Netherlands Consortium for Healthy Ageing, Leiden, the Netherlands); Elizabeth G. Holliday, PhD (Hunter Medical Research Institute and Faculty of Health, University of Newcastle, Newcastle, NSW, Australia) Jennifer Huffman, PhD (MRC Human Genetics Unit, Institute of Genetics and Molecular Medicine, University of Edinburgh, Edinburgh, UK); Sharon L. R. Kardia, PhD (Department of Epidemiology, University of Michigan, Ann Arbor); Nicole Kochan, PhD (Centre for Healthy Brain Ageing, School of Psychiatry, University of New South Wales, Sydney, NSW, Australia; Neuropsychiatric Institute, Prince of Wales Hospital, Sydney, NSW, Australia); David S. Knopman, MD (Department of Neurology, Mayo Clinic, Rochester, MN); John B. Kwok, PhD (Neuroscience Research Australia, Randwick, NSW, Australia; School of Medical Sciences, University of New South Wales, Sydney, NSW, Australia) Jean-Charles Lambert, PhD (Inserm-UMR744, Institut Pasteur de Lille, Unité d'Epidémiologie et de Santé Publique, Lille, France); Teresa Lee, PhD (Centre for Healthy Brain Ageing, School of
Psychiatry, University of New South Wales, Sydney, NSW, Australia; Neuropsychiatric Institute, Prince of Wales Hospital, Sydney, NSW, Australia); Guo Li, MS (Cardiovascular Health Research Unit, Department of Medicine, University of Washington, Seattle); Shu-Chen Li, PhD (Max Planck Institute for Human Development, Berlin, Germany; Technische Universität Dresden, Dresden, Germany); Marisa Loitfelder, PhD (Department of Neurology, Medica University of Graz, Graz, Austria); Oscar L. Lopez, MD (Department of Neurology, University of Pittsburgh, Pittsburgh, PA); Astri J. Lundervold, PhD (Department of Biological and Medical Psychology, University of Bergen, Bergen, Norway; Kavli Research Centre for Aging and Dementia, Haraldsplass Deaconess Hospital, Bergen, Norway; K.G. Jebsen Centre for Research on Neuropsychiatric Disorders, University of Bergen, Bergen, Norway); Anders Lundqvist, PhD (Umeå Center for Functional Brain Imaging [UFBI], Umeå University, Umeå, Sweden); Karen A. Mather, PhD (Centre for Healthy Brain Ageing, School of Psychiatry, University of New South Wales, Sydney, NSW, Australia); Saira S. Mirza, MD (Department of Epidemiology, Erasmus University Medical Center, Rotterdam, the Netherlands; Netherlands Consortium for Healthy Ageing, Leiden, the Netherlands); Lars Nyberg, PhD (Umeå Center for Functional Brain Imaging [UFBI], Umeå University, Umeå, Sweden; Department of Radiation Sciences, Umeå University, Umeå, Sweden; Department of Integrative Medical Biology, Umeå University, Umeå, Sweden); Ben A. Oostra, PhD (Genetic

Epidemiology Unit, Department of Epidemiology, Erasmus University Medical Center, Rotterdam, the Netherlands); Aarno Palotie, MD, PhD (Wellcome Trust Sanger Institute, Wellcome Trust Genome Campus, Cambridge, UK; Institute for Molecular Medicine Finland [FIMM], University of Helsinki, Helsinki, Finland; Department of Medical Genetics, University of Helsinki and University Central Hospital, Helsinki, Finland); Goran Papenberg, PhD (Max Planck Institute for Human Development, Berlin, Germany; Karolinska Institutet, Aging Research Center, Stockholm University, Stockholm, Sweden); Allison Pattie, BSc (Department of Psychology, University of Edinburgh, Edinburgh, UK); Katja Petrovic, MSc (Department of Neurology, Medical University of Graz, Graz, Austria); Ozren Polasek, MD, PhD (Faculty of Medicine, Department of Public Health, University of Split, Split, Croatia); Bruce M. Psaty, MD, PhD (Cardiovascular Health Research Unit, Department of Medicine, University of Washington, Seattle; Deparment of Epidemiology, University of Washington, Seattle; Deparment of Health Services, University of Washington, Seattle; Group Health Research Unit, Group Health Cooperative, Seattle, WA); Paul Redmond, MSc (Department of Psychology, University of Edinburgh, Edinburgh, UK); Simone Reppermund, PhD (Centre for Healthy Brain Ageing, School of Psychiatry, University of New South Wales, Sydney, NSW, Australia); Jerome I. Rotter, MD (Institute for Translational Genomics and Population Sciences, Los Angeles BioMedical Research Institute, Harbor-UCLA Medical Center, Los Angeles, CA; Division of Genetic Outcomes, Department of Pediatrics, Harbor-UCLA Medical Center, Los Angeles, CA); Helena Schmidt, MD, PhD (Department of Neurology, Medical University of Graz, Graz, Austria; Centre for Molecular Medicine, Institute of Molecular Biology and Biochemistry, Medical University of Graz, Graz, Austria); Maaike Schuur, PhD (Department of Neurology, Erasmus University Medical Center, Rotterdam, the Netherlands; Genetic Epidemiology Unit, Department of Epidemiology, Erasmus University 
Medical Center, Rotterdam, the Netherlands); Peter W. Schofield, MD (School of Medicine and Public Health, University of Newcastle, Newcastle, NSW, Australia); Rodney J. Scott, PhD (Hunter Medical Research Institute and Faculty of Health, University of Newcastle, Newcastle, NSW, Australia); Vidar M. Steen, PhD (K.G. Jebsen Centre for Psychosis Research and the Norwegian Centre for Mental Disorders Research [NORMENT], Department of Clinical Science, University of Bergen, Bergen, Norway; Dr Einar Martens Research Group for Biological Psychiatry, Center for Medical Genetics and Molecular Medicine, Haukeland University Hospital, Bergen, Norway); David J. Stott, MD, PhD (Institute of Cardiovascular and Medical Sciences, University of Glasgow, Glasgow, UK); John C. van Swieten, MD, PhD (Department of Neurology, Erasmus University Medical Center, Rotterdam, the Netherlands); Kent D. Taylor, PhD (Institute for Translational Genomics and Population Sciences, Los Angeles BioMedical Research Institute, Harbor-UCLA Medical Center, Los Angeles, CA; Department of Pediatrics, Harbor-UCLA Medical Center, Los Angeles, CA); Julian Trollor, MD (Centre for Healthy Brain Ageing, School of Psychiatry, University of New South Wales, Sydney, NSW, Australia; Department of Developmental Disability Neuropsychiatry, School of Psychiatry, University of New South Wales, Sydney, NSW, Australia); Stella Trompet, PhD (Department of Gerontology and Geriatrics, Leiden University Medical Center, Leiden, the Netherlands; Department of Cardiology, Leiden University Medical Center, Leiden, the Netherlands); Andre G. Uitterlinden, PhD (Department of Epidemiology, Erasmus University Medical Center, Rotterdam, the Netherlands; Netherlands Consortium for Healthy Ageing, Leiden, the Netherlands; Department of Internal Medicine, Erasmus University Medical Center, Rotterdam, the Netherlands); Galit Weinstein, PhD (Department of Neurology, Boston University School of Medicine, Boston, MA; Framingham Heart Study, Framingham, MA); Elisabeth Widen, MD, PhD (Institute for Molecular Medicine Finland [FIMM], University of Helsinki, Helsinki, Finland); Beverly G. Windham, MD (Division of Geriatrics, Department of Medicine University of Mississippi Medical Center, Jackson); J. W. Jukema, MD, PhD (Department of Cardiology, Leiden University Medical Center, Leiden, the Netherlands; Durrer Center for Cardiogenetic Research, Amsterdam, the Netherlands; Interuniversity Cardiology Institute of the Netherlands, Utrecht, the Netherlands); Alan F. Wright, PhD (MRC Human Genetics Unit, Institute of Genetics and Molecular Medicine, University of Edinburgh, Edinburgh, UK); Margaret J. Wright, PhD (Neuroimaging Genetics Group, QIMR Berghofer Medical Research Institute, Brisbane, QLD, Australia); Qiong Yang, PhD (Framingham Heart Study, Framingham, MA; Department of Biostatistics, Boston University School of Public Health, Boston, MA); Helene Amieva, PhD (Inserm U897, Université Bordeaux Segalen, Bordeaux, France); John R. Attia, PhD (Hunter Medical Research Institute and Faculty of Health, University of Newcastle, Newcastle, NSW, Australia); David A. Bennett, MD (Rush Alzheimer's Disease Center, Rush University Medical Center, Chicago, IL); Henry Brodaty, MD, DSc (Centre for Healthy Brain Ageing. School of Psychiatry, University of New South Wales, Sydney, NSW, Australia; Dementia Collaborative Research Centre, University of New South Wales, Sydney, NSW, Australia); Anton J. M. de Craen, PhD (Department of Gerontology and Geriatrics, Leiden University Medical Center, Leiden, the Netherlands; Netherlands Consortium for Healthy Ageing, Leiden, the Netherlands); Caroline
Hayward, PhD (MRC Human Genetics Unit, Institute of Genetics and Molecular Medicine, University of Edinburgh, Edinburgh, UK); M. Arfan Ikram, MD, PhD (Department of Neurology, Erasmus University Medical Center, Rotterdam, the Netherlands; Department of Epidemiology, Erasmus University Medical Center, Rotterdam, the Netherlands; Netherlands Consortium for Healthy Ageing, Leiden, the Netherlands; Department of Radiology, Erasmus University Medical Center, Rotterdam, the Netherlands); Ulman Lindenberger, PhD (Max Planck Institute for Human Development, Berlin, Germany); Lars-Goran Nilsson, PhD (ARC, Karolinska Institutet, Stockholm and UFBI, Umeå University, Umeå, Sweden); David J. Porteous, PhD (Centre for Cognitive Ageing and Cognitive Epidemiology, University of Edinburgh, Edinburgh, UK; Medical Genetics Section, University of Edinburgh Centre for Genomic and Experimental Medicine, Institute of Genetics and Molecular Medicine, Western General Hospital, Edinburgh, UK; Generation Scotland, University of Edinburgh Centre for Genomic and Experimental Medicine, Institute of Genetics and Molecular Medicine, Western General Hospital, Edinburgh, UK); Katri Räikkönen, PhD (Institute of Behavioural Sciences, University of Helsinki, Helsinki, Finland); Ivar Reinvang, $\mathrm{PhD}$ (Department of Psychology, University of Oslo, Oslo, Norway); Igor Rudan, MD, PhD (Centre for Population Health Sciences, University of Edinburgh, Edinburgh, UK); Perminder S. Sachdev, MD, PhD (Centre for Healthy Brain Ageing, School of Psychiatry, University of New South Wales, Sydney, NSW, Australia; Neuropsychiatric Institute, Prince of Wales Hospital, Sydney, NSW, Australia); Reinhold Schmidt, MD (Department of Neurology, Medical University of Graz, Graz, Austria); Peter R. Schofield, PhD (Neuroscience Research Australia, Sydney, NSW Australia; Faculty of Medicine, University of New South Wales, Sydney, NSW, Australia); Velandai Srikanth, PhD (Menzies Research Institute, Hobart, Tasmania; Stroke and Ageing Research, Medicine, Southern Clinical School, Monash University, Melbourne, VIC, Australi); John M. Starr, PhD (Centre for Cognitive Ageing and Cognitive Epidemiology, University of Edinburgh, Edinburgh, UK; Alzheimer Scotland Dementia Research Centre, University of Edinburgh, Edinburgh, UK); Stephen T. Turner, MD (Division of Nephrology and Hypertension Department of Internal Medicine, Mayo Clinic, Rochester, MN); David R. Weir, PhD (Survey Research Center, Institute for Social Research, University of Michigan, Ann Arbor); James F. Wilson, DPhil (Centre for Population Health Sciences, University of Edinburgh, Edinburgh, UK); Cornelia M. van Duijn, PhD (Genetic Epidemiology Unit, Department of Epidemiology, Erasmus University Medical Center, Rotterdam, the Netherlands; Netherlands Consortium for Healthy Ageing, Leiden, the Netherlands); Lenore J. Launer, PhD (Intramural Research Program National Institutes on Aging National Institutes of Health, Bethesda, MD); Annette L. Fitzpatrick, PhD (Deparment of Epidemiology, University of Washington, Seattle; Department of Global Health, University of Washington, Seattle); Sudha Seshadri, MD (Department of Neurology, Boston University School of Medicine, Boston, MA; Framingham Heart Study, Framingham, MA); Thomas H. Mosley Jr, PhD (Division of Geriatrics, Department of Medicine, University of Mississippi Medical Center, Jackson); and lan J. Deary, PhD (Centre for Cognitive Ageing and Cognitive Epidemiology, University of Edinburgh, Edinburgh, UK; Department of Psychology, University of Edinburgh, Edinburgh, UK)
Additional Contributions: We thank all study participants and the researchers involved in the Schizophrenia Working Group of the Psychiatric Genomics Consortium (http://www.med.unc.edu (pgc), Psychiatric Genomics Consortium, Cohorts for Heart and Aging Research in Genomic Epidemiology, the Cognitive Genomics Consortium, and the UK Biobank for their contributions.

\section{REFERENCES}

1. Whiteford HA, Degenhardt L, Rehm J, et al. Global burden of disease attributable to mental and substance use disorders: findings from the Global Burden of Disease Study 2010. Lancet. 2013;382 (9904):1575-1586.

2. Green MF, Harvey PD. Cognition in schizophrenia: past, present, and future. Schizophr Res Cogn. 2014;1(1):e1-e9.

3. Green MF, Kern RS, Braff DL, Mintz J. Neurocognitive deficits and functional outcome in schizophrenia: are we measuring the "right stuff"? Schizophr Bull. 2000;26(1):119-136.

4. Kahn RS, Keefe RS. Schizophrenia is a cognitive illness: time for a change in focus. JAMA Psychiatry. 2013;70(10):1107-1112.

5. Dickinson D, Ragland JD, Gold JM, Gur RC. General and specific cognitive deficits in schizophrenia: Goliath defeats David? Biol Psychiatry. 2008;64(9):823-827.

6. Aas M, Dazzan P, Mondelli V, Melle I, Murray RM, Pariante CM. A systematic review of cognitive function in first-episode psychosis, including a discussion on childhood trauma, stress, and inflammation. Front Psychiatry. 2014;4:182.

7. Reichenberg A, Caspi A, Harrington $\mathrm{H}$, et al. Static and dynamic cognitive deficits in childhood preceding adult schizophrenia: a 30-year study. Am J Psychiatry. 2010;167(2):160-169.

8. Fusar-Poli P, Deste G, Smieskova R, et al. Cognitive functioning in prodromal psychosis: a meta-analysis. Arch Gen Psychiatry. 2012;69(6):562-571.

9. Mohamed S, Rosenheck R, Swartz M, StroupS, Lieberman JA, Keefe RS. Relationship of cognition and psychopathology to functional impairment in schizophrenia. Am J Psychiatry. 2008;165(8):978-987.

10. Keefe RS, Buchanan RW, Marder SR, et al. Clinical trials of potential cognitive-enhancing drugs in schizophrenia: what have we learned so far? Schizophr Bull. 2013;39(2):417-435.

11. Zai G, Robbins TW, Sahakian BJ, Kennedy JL. A review of molecular genetic studies of neurocognitive deficits in schizophrenia. Neurosci Biobehav Rev. 2017;72:50-67.

12. Keshavan MS, KulkarniS, Bhojraj T, et al. Premorbid cognitive deficits in young relatives of schizophrenia patients. Front Hum Neurosci. 2010;3:62.

13. McIntosh AM, Harrison LK, Forrester K, Lawrie SM, Johnstone EC. Neuropsychological impairments in people with schizophrenia or bipolar disorder and their unaffected relatives. $\mathrm{Br} J$ Psychiatry. 2005;186:378-385.

14. Bora E, Lin A, Wood SJ, Yung AR, McGorry PD, Pantelis C. Cognitive deficits in youth with familial and clinical high risk to psychosis: a systematic review and meta-analysis. Acta Psychiatr Scand. 2014;130(1):1-15.

15. Toulopoulou T, Goldberg TE, Mesa IR, et al. Impaired intellect and memory: a missing link 
between genetic risk and schizophrenia? Arch Gen Psychiatry. 2010;67(9):905-913.

16. Fowler T, Zammit S, Owen MJ, Rasmussen F. A population-based study of shared genetic variation between premorbid IQ and psychosis among male twin pairs and sibling pairs from Sweden. Arch Gen Psychiatry. 2012;69(5):460-466.

17. McIntosh AM, Gow A, Luciano M, et al. Polygenic risk for schizophrenia is associated with cognitive change between childhood and old age. Biol Psychiatry. 2013;73(10):938-943.

18. Lencz T, Knowles E, Davies G, et al. Molecular genetic evidence for overlap between general cognitive ability and risk for schizophrenia: a report from the Cognitive Genomics Consortium (COGENT). Mol Psychiatry. 2014;19(2):168-174.

19. Hubbard L, Tansey KE, Rai D, et al. Evidence of common genetic overlap between schizophrenia and cognition. Schizophr Bull. 2016;42(3):832-842.

20. Liebers DT, Pirooznia M, Seiffudin F, Musliner $\mathrm{KL}$, Zandi PP, Goes FS. Polygenic risk of schizophrenia and cognition in a population-based survey of older adults. Schizophr Bull. 2016;42(4): 984-991.

21. Hatzimanolis $A$, Bhatnagar $P$, Moes $A$, et al. Common genetic variation and schizophrenia polygenic risk influence neurocognitive performance in young adulthood. Am J Med Genet B Neuropsychiatr Genet. 2015;168B(5):392-401.

22. Hagenaars SP, Harris SE, Davies G, et al; METASTROKE Consortium, International Consortium for Blood Pressure GWAS; SpiroMeta Consortium; CHARGE Consortium Pulmonary Group, CHARGE Consortium Aging and Longevity Group. Shared genetic aetiology between cognitive functions and physical and mental health in UK Biobank ( $\mathrm{N}=112151)$ and 24 GWAS consortia. $\mathrm{Mol}$ Psychiatry. 2016;21(11):1624-1632.

23. Hill WD, Davies G, Liewald DC, McIntosh AM, Deary IJ; CHARGE Cognitive Working Group. Age-dependent pleiotropy between general cognitive function and major psychiatric disorders. Biol Psychiatry. 2016;80(4):266-273.

24. Trampush JW, Yang ML, Yu J, et al. GWAS meta-analysis reveals novel loci and genetic correlates for general cognitive function: a report from the COGENT consortium. Mol Psychiatry. 2017;22(3):336-345.

25. Ripke S, O'Dushlaine C, Chambert K, et al; Multicenter Genetic Studies of Schizophrenia Consortium; Psychosis Endophenotypes International Consortium; Wellcome Trust Case Control Consortium 2. Genome-wide association analysis identifies 13 new risk loci for schizophrenia. Nat Genet. 2013;45(10):1150-1159.

26. Davies G, Armstrong N, Bis JC, et al; Generation Scotland. Genetic contributions to variation in general cognitive function: a meta-analysis of genome-wide association studies in the CHARGE consortium ( $N$ = 53 949). Mol Psychiatry. 2015;20 (2):183-192.

27. Davies G, Marioni RE, Liewald DC, et al. Genome-wide association study of cognitive functions and educational attainment in UK Biobank ( $=112$ 151). Mol Psychiatry. 2016;21(6): 758-767.

28. Schizophrenia Working Group of the Psychiatric Genomics Consortium. Biological insights from 108 schizophrenia-associated genetic loci. Nature. 2014;511(7510):421-427.

29. Liu JZ, Hov JR, Folseraas T, et al; UK-PSCSC Consortium; International PSC Study Group; International IBD Genetics Consortium. Dense genotyping of immune-related disease regions identifies nine new risk loci for primary sclerosing cholangitis. Nat Genet. 2013;45(6):670-675.

30. Schork AJ, Wang Y, Thompson WK, Dale AM, Andreassen OA. New statistical approaches exploit the polygenic architecture of schizophreniaimplications for the underlying neurobiology. Curr Opin Neurobiol. 2016;36:89-98.

31. Andreassen OA, Thompson WK, Dale AM. Boosting the power of schizophrenia genetics by leveraging new statistical tools. Schizophr Bull. 2014;40(1):13-17.

32. Andreassen OA, Thompson WK, Schork AJ et al; Psychiatric Genomics Consortium (PGC); Bipolar Disorder and Schizophrenia Working Groups. Improved detection of common variants associated with schizophrenia and bipolar disorder using pleiotropy-informed conditional false discovery rate. PLoS Genet. 2013;9(4):e1003455.

33. Andreassen OA, Harbo HF, Wang Y, et al; Psychiatric Genomics Consortium (PGC) Bipolar Disorder and Schizophrenia Work Groups; International Multiple Sclerosis Genetics Consortium (IMSGC). Genetic pleiotropy between multiple sclerosis and schizophrenia but not bipolar disorder: implications for immune-related gene loci. Mol Psychiatry. 2015;20(2):207-214.

34. Andreassen OA, Djurovic S, Thompson WK, et al; International Consortium for Blood Pressure GWAS; Diabetes Genetics Replication and Meta-analysis Consortium; Psychiatric Genomics Consortium Schizophrenia Working Group. Improved detection of common variants associated with schizophrenia by leveraging pleiotropy with cardiovascular-disease risk factors. Am J Hum Genet. 2013;92(2):197-209.

35. Le Hellard S, Wang Y, Witoelar A, et al; Schizophrenia Working Group of the Psychiatric Genomics Consortium. Identification of gene loci that overlap between schizophrenia and educational attainment. Schizophr Bull. 2017;43(3): 654-664.

36. Desikan RS, Schork AJ, Wang Y, et al; Inflammation Working Group and International Genomics of Alzheimer's Disease Project (IGAP) and DemGene Investigators. Polygenic overlap between c-reactive protein, plasma lipids, and alzheimer disease. Circulation. 2015;131(23):2061 2069.

37. Andreassen OA, Desikan RS, Wang Y, et al. Abundant genetic overlap between blood lipids and immune-mediated diseases indicates shared molecular genetic mechanisms [published correction appears in PLoS One. 2015;10(5): e0128048]. PLoS One. 2015;10(4):e0123057.

38. Nichols T, Brett M, Andersson J, Wager T, Poline JB. Valid conjunction inference with the minimum statistic. Neuroimage. 2005;25(3):653-660.

39. Schwartzman A, Lin X. The effect of correlation in false discovery rate estimation. Biometrika. 2011; 98(1):199-214.

40. Kang HJ, Kawasawa $\mathrm{Yl}$, Cheng F, et al. Spatio-temporal transcriptome of the human brain. Nature. 2011;478(7370):483-489.
41. Trabzuni $D$, Ryten $M$, Walker R, et al. Quality control parameters on a large dataset of regionally dissected human control brains for whole genome expression studies. J Neurochem. 2011;119(2):275-282.

42. GTEx Consortium. The Genotype-Tissue Expression (GTEx) project. Nat Genet. 2013;45(6): 580-585.

43. Pers TH, Karjalainen JM, Chan Y, et al; Genetic Investigation of ANthropometric Traits (GIANT) Consortium. Biological interpretation of genome-wide association studies using predicted gene functions. Nat Commun. 2015;6:5890.

44. Sniekers $S$, Stringer $S$, Watanabe $K$, et al. Genome-wide association meta-analysis of 78,308 individuals identifies new loci and genes influencing human intelligence [published online May 22, 2017]. Nat Genet. doi:10.1038/ng.3869

45. Nardini M, Gnesutta N, Donati G, et al. Sequence-specific transcription factor NF-Y displays histone-like DNA binding and H2B-like ubiquitination. Cell. 2013;152(1-2):132-143.

46. Yamanaka T, Tosaki A, Kurosawa M, et al. NF-Y inactivation causes atypical neurodegeneration characterized by ubiquitin and p62 accumulation and endoplasmic reticulum disorganization. Nat Commun. 2014;5:3354.

47. Power RA, Steinberg S, Bjornsdottir G, et al. Polygenic risk scores for schizophrenia and bipolar disorder predict creativity. Nat Neurosci. 2015;18(7): 953-955.

48. Palmer BW, Heaton RK, Paulsen JS, et al. Is it possible to be schizophrenic yet neuropsychologically normal? Neuropsychology. 1997;11(3):437-446.

49. Babbs C, Lloyd D, Pagnamenta AT, et al; International Molecular Genetic Study of Autism Consortium (IMGSAC). De novo and rare inherited mutations implicate the transcriptional coregulator TCF2O/SPBP in autism spectrum disorder. J Med Genet. 2014;51(11):737-747.

50. Clark NE, Garman SC. The 1.9 Å structure of human a- $\mathrm{N}$-acetylgalactosaminidase: the molecular basis of Schindler and Kanzaki diseases. J Mol Biol. 2009;393(2):435-447.

51. Ferguson CS, Tyndale RF. Cytochrome P450 enzymes in the brain: emerging evidence of biological significance. Trends Pharmacol Sci. 2011; 32(12):708-714.

52. Schyman P, Lai W, Chen H, Wang Y, Shaik S. The directive of the protein: how does cytochrome P450 select the mechanism of dopamine formation? J Am Chem Soc. 2011;133(20):7977-7984.

53. Devor A, Andreassen OA, Wang Y, et al. Genetic evidence for role of integration of fast and slow neurotransmission in schizophrenia. Mol Psychiatry. 2017;22(6):792-801.

54. Zhang $Q$, Shen $Q, X u Z$, et al. The effects of CACNA1C gene polymorphism on spatial working memory in both healthy controls and patients with schizophrenia or bipolar disorder. Neuropsychopharmacology. 2012;37(3):677-684.

55. Dietsche B, Backes $H$, Laneri $D$, et al. The impact of a CACNA1C gene polymorphism on learning and hippocampal formation in healthy individuals: a diffusion tensor imaging study. Neuroimage. 2014;89:256-261.

56. Solovieff N, Cotsapas C, Lee PH, Purcell SM, Smoller JW. Pleiotropy in complex traits: challenges and strategies. Nat Rev Genet. 2013;14(7):483-495. 